\title{
Case Report: Gyral Brain Arteriovenous Malformation with Presentation Isolated from Intraventricular Hemorrhage
}

\author{
Rizky Fawzyana Sarengat ${ }^{*}$, Achmad Firdaus Sani²
}

\author{
${ }^{1}$ Neurology Resident of Medical Faculty Airlangga University and Dr.Soetomo General \\ Hospital Surabaya Indonesia
}
${ }^{2}$ Staff of Neuroimajing Division at Neurology Departement of Medical Faculty Airlangga University and Dr.Soetomo General Hospital Surabaya Indonesia

\section{*Corresponding author details: Rizky Fawzyana Sarengat; rizkyfawzyana@gmail.com}

\begin{abstract}
Introduction: Brain Arteriovenous Malformation (BAVM) is a rare disease with an incidence rate of 1.3 out of 100,000 people. The most common clinical manifestations in BAVM patients are intracranial hemorrhage. There were $38.5 \%$ of BAVM patients who had intraventricular hemorrhage (IVH), and the rate of IVH without parenchymal bleeding in the ruptured AVM is $7.8 \%$. We reported a case of BAVM with intraventricular hemorrhage presentation without intracerebral hemorrhage (isolated IVH). Case: Male, 15 years old, with a chief complaint of acute decreased consciousness on activity, followed by vomiting and general onset seizures. There was no history of hypertension or trauma before. From the neurological examination, the GCS is 225 , and there were positive pathological reflexes on both sides. From a head CT scan without contrast, we found IVH in bilateral lateral ventricles, ventricle III and ventricle IV, communicating hydrocephalus, and brain edema. Head and Neck Computed Tomography Angiography (CTA) found a compact AVM in the left centrum semiovalecortex parietal (Spetzler Martin AVM grading system grade 3). Patients underwent EVD surgery for hydrocephalus. The following steps are cerebral DSA as the gold standard for AVM diagnostic and targeted embolization to prevent further bleeding. Conclusion: One of the causes of Isolated Intraventricular Hemorrhage (IIVH) is AVM. In this case, although the topography of AVM is in the gyral, it can cause IIVH manifestation if there is contact between the AVM nidus and the ventricle.
\end{abstract}

Keywords: arteriovenous malformation; intraventricular hemorrhage; isolated intraventricular hemorrhage

\section{INTRODUCTION}

Brain Arterio Venous Malformation (BAVM) is a vascular lesion consisting of an abnormal network of blood vessels (nidus) with shunting of pathological blood flow from the arteries to the veins without involving the regular capillary bed. ${ }^{1}$ The incidence of BAVM is relatively rare, but it can cause severe or irreversible neurologic symptoms and even causes death. The incidence rate of BAVM is 1.3 out of 100,000 people. ${ }^{2}$ BAVM often affects young adults, with the most common age at diagnosis ranging from 20 to 40 years with no sex predilection. ${ }^{3}$

The ArterioVenous Malformation (AVM) structure consists of feeding arteries that flow directly to the draining veins through one or more fistulas without involving the capillary bed. ${ }^{3}$ One of the topographical classifications of AVM is the gyral AVM locating within a specific gyrus covered by the cortex. The large gyral AVM expands the gyrus, compresses the adjacent sulcus, and extends into the subcortical white matter and the ventricle wall. ${ }^{4}$ Intraventricular Hemorrhage (IVH) is a common complication of intracerebral hemorrhage and subarachnoid hemorrhage associated with poor functional outcomes. ${ }^{3}$ Extension of bleeding into the ventricles is common in ruptured AVM. Case reports of IVH in AVM have previously also been reported. 5 As many as $38.5 \%$ of patients with ruptured BAVM had IVH. ${ }^{3}$ Isolated IVH (IIVH) in adults is rare, accounting for $3 \%$ of the incidence of intracerebral hemorrhage. ${ }^{2}$
AVM is clinically asymptomatic in $15 \%$ of cases until onset. Approximately $41 \%$ - $79 \%$ have a clinical intracranial hemorrhage, which is more common in children. Symptoms caused by bleeding are loss of consciousness, sudden and severe headache, nausea, and vomiting. Sequelae due to damage to local brain tissue at the bleeding site include seizures, hemiparesis, sensory loss on one side of the body and language deficits.

BAVM is first identified on cross-sectional imaging, i.e., computed tomography (CT) or magnetic resonance imaging (MRI). On non-contrast CT, the nidus is seen as a slightly hyperdense blood density compared to adjacent brain tissue. On Computed Tomography Angiography (CTA), a clear picture of the feeding arteries, nidus, and draining veins will appear called the "bag of a worm." Angiography remains the gold standard for diagnosis and treatment planning of AVM. (3) The three components of AVM that can be seen from digital subtraction angiography (DSA) are enlarged feeding arteries, nidus of congested vessels, and dilated draining veins.

The definitive treatment for BAVM is by removing the nidus and arteriovenous shunt. This therapy usually involves endovascular embolization, microsurgical resection, and stereotactic radiosurgery. Embolization itself divides into curative, preoperative or preradiosurgery, targeted, and palliative embolization. 
Targeted embolization aims to close the lesion having the weakest angioarchitecture (weakest point), which is the source of bleeding, for example, in BAVM patients with perinidal aneurysms who have manifestations of subarachnoid hemorrhage. Appropriate embolization or occlusion at the aneurysm or weakest point will restore the natural course of the unruptured BAVM. ${ }^{7}$

\section{CASE}

A 15-year-old man with the chief complaint of a sudden loss of consciousness on activity followed by general-onset vomiting and seizures came to the doctor. The patient had previously complained of headaches. The patient had no history of seizures. There was no history of fever or previous trauma, and he also denied that he had hypertension history, stroke, tumour, brain infection, and anticoagulant/antiplatelet drugs usage.

The vital sign was found within normal limits on physical examination. GCS 225 was found on neurological examination, and no neck stiffness and meningeal sign were found. Cranial nerve examination was within normal limits. No lateralization was obtained on motor examination. Sensory disturbances were unable to evaluate because of decreased consciousness. Physiological reflexes are within normal limits. Babinsky and Chaddock's pathological reflexes were positive on both sides. Autonomic and vertebral column examination within normal limits.

Laboratory examinations showed normal results on complete blood count and clinical chemistry examination. Chest X-Ray and ECG examinations were within normal limits. A CT scan of the head without contrast revealed IVH in bilateral lateral ventricles, ventricles III and IV, as well as communicating hydrocephalus and brain edema (Figure 1). There was no evidence of intraparenchymal hemorrhage. The head and neck CTA examination revealed a worm-like appearance with multiple calcifications leading to an AVM with a nidus size of $\pm 3.2 \mathrm{~cm} \times 2.2 \mathrm{~cm} \times 3.4 \mathrm{~cm}$ in the central of semiovale-cortex left parietal lobe and received a feeding artery from the left anterior cerebral artery branches (A2 and A3), draining veins to the superior sagital sinus and Trolard's superior anastomotic vein (Spetzler-Martin Grade III Arteriovenous Malformation). Neither flow-related aneurysm nor intranidal aneurysm was seen on CTA (Fig. 2).

Subsequently, DSA and targeted embolization were carried out as an advanced stage of AVM management. An intranidal aneurysm from the cerebral DSA examination was seen at the edge of the lateral ventricle, which was not visible on the CTA examination. The first stage of embolization was performed, targeting the source of bleeding (intranidal aneurysm). After embolization, the nidus was reduced by $50 \%$. The patient was advised for the second stage of embolization in 1-2 months.

\section{DISCUSSION}

BAVM is a rare pathology. Intracranial hemorrhage is a common and severe manifestation of BAVM. Intracranial hemorrhage in this context includes parenchymal hemorrhage and intraventricular hemorrhage. The etiology of IVH is multiple, including hypertensive intracerebral hemorrhage (ICH), intracranial aneurysm rupture, AVM rupture, and trauma. 8

IVH is frequently found to be associated with intraparenchymal or subarachnoid hemorrhage, whereas isolated IVH (IIVH) is rare in adults, accounting for only $3 \%$ of all intracerebral hemorrhages and is rarely described. IVH coexisting with parenchymal hemorrhage is always caused by an AVM located deeper or closer to the ventricular wall. 2,9 It is essential to evaluate the possible causes of IIVH in patient management. Many etiologies remain possible causes of IIVH even after the exclusion of the traumatic reason. AVM and aneurysms are the most common causes, but other vascular causes should be sought, including cavernous malformations and Moyamoya disease. Arterial hypertension, use of anticoagulants drugs, coagulopathy, and certain toxic substances are also associated with IIVH. 10

As many as $38.5 \%$ of ruptured BAVM patients had IVH, and the incidence of IVH without parenchymal hemorrhage in ruptured BAVM patients was only 7.8\%.6 AVM in IIVH cases can be divided into intraventricular or extraventricular. The intraventricular AVM (from the choroid plexus) is often small and maybe hidden angiographically. It can cause IIVH but with massive bleeding that extends into the adjacent cerebral parenchyma. Extraventricular AVM can cause IIVH through the nidus contact with the ventricles or by angioarchital weak points such as flow-related aneurysms, venous ectasia, or deep venous drainage. More minor and deeper AVM may be more prone to rupture and rebleeding. 10

In a reported case, a 15-year-old man came to Emergency Ward with the chief complaint of a sudden loss of consciousness occurring during activity, and it was followed by vomiting, general onset seizures, and previous history of headache. Neurological examination revealed a GCS of 225, and it also revealed Babinski and Chaddock's pathological reflexes were positive on both sides.

CT scan of the head without contrast and CTA of the head and neck found a worm-like appearance with multiple classifications supporting of AVM in the central of semiovale-cortex left parietal lobe and a Spetzler-Martin Grade III Arteriovenous Malformation and IVH in the bilateral lateral ventricles, ventricles III and IV, communicating hydrocephalus, and brain edema. There was no evidence of intraparenchymal hemorrhage. Patient's symptoms consist of decreased consciousness and general onset seizures. These symptoms can be caused by intraventricular hemorrhage and brain edema.

Although the AVM location is in the gyrus topographically, which supposed to show intraparenchymal hemorrhage, but in this patient's manifestation IVH is isolated. This case can be explained due to the AVM's nidus in contact with the ventricle. DSA is our consideration for further action, as the standard to see in more detail the architecture of the internal blood vessels of the AVM.

\section{CONCLUSION}

One of the causes of isolated intraventricular hemorrhage (IIVH) is AVM. In this case, the topography of the AVM located in the gyrus can cause IIVH manifestations if there is contact between the AVM nidus and the ventricles.

\section{REFERENCES}

[1] Bryne, Vincent J. Tutorials in endovascular neurosurgery and interventional neuroradiology. New York: Springer-Verlag Berlin Heidelberg; 2012.

[2] Barreaue X, Marnat G, Gariel F, Dousset V. Intracranial arteriovenous malformations. Diagn Interv Imagin. 2014;95(12):1175-86. doi: $10.1016 /$ j.diii.2014.10.004

[3] Bokhari MR, Bokhari SRA. Arteriovenous malformation of the brain. StatPearls Publishing; 2020.

[4] Tanaka, Michiro. AVM Definition and angioarchitecture. In: Brain arteriovenous malformation. Springer International Publishing; 2017. p. 5-22. 
[5] Kurniawan D, Ardhi MS. Perdarahan intraventrikel sebagai manisfestasi utama dari ruptur malformasi arteri vena fossa posterior. Aksona. 2019;1(4):96-101.

[6] Osborn AG, Digre KB. Arterivenous malformation. In: Imagin in neurologi. 1st ed. Salt Lake City: Elsevier Inc.; 2016. p. 14-22.

[7] Sani A, Putri S, Usman F. Brain arteriovenous malformation. In: Konsensus nasional neurointervensi. Airlangga University Press; 2020. p. 23.
[8] Gilard V, Metayer T, Langlois CGO, Proust F, Emery E, Gaberel T. Intraventricular hemorrhage related to AVM rupture: Description, outcomes and impact of intraventricular fibrinolysis. Clin Neurol Neurosurg. 2018; 164:92-6.

[9] Ye Z, Ai X, Hu X, Fang F, You C. Clinical features and prognostic factors in patients with intraventricular hemorrhage caused by ruptured arteriovenous malformations. Medicine. 2017;96(45): e8544.

[10] Barnaure I, Liberato A, Gonzalez R, Romero J. Isolated intraventricular haemorrhage in adults. Br J Radiol. 2016;90(1069).

\section{APPENDIX}
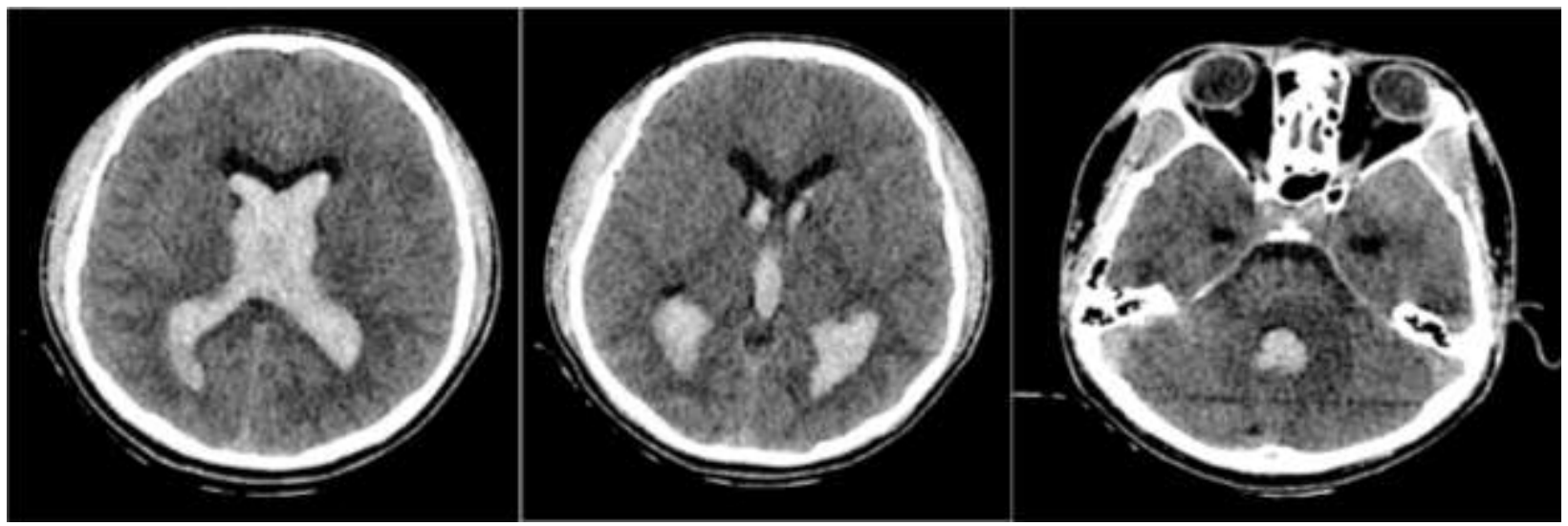

FIGURE 1: (A) CT scan of the head without contrast with axial view shows communicating hydrocephalus, brain edema, and IVH in the bilateral lateral ventricles. (B) IVH in the ventricles III. (C) IVH in the ventricle IV.
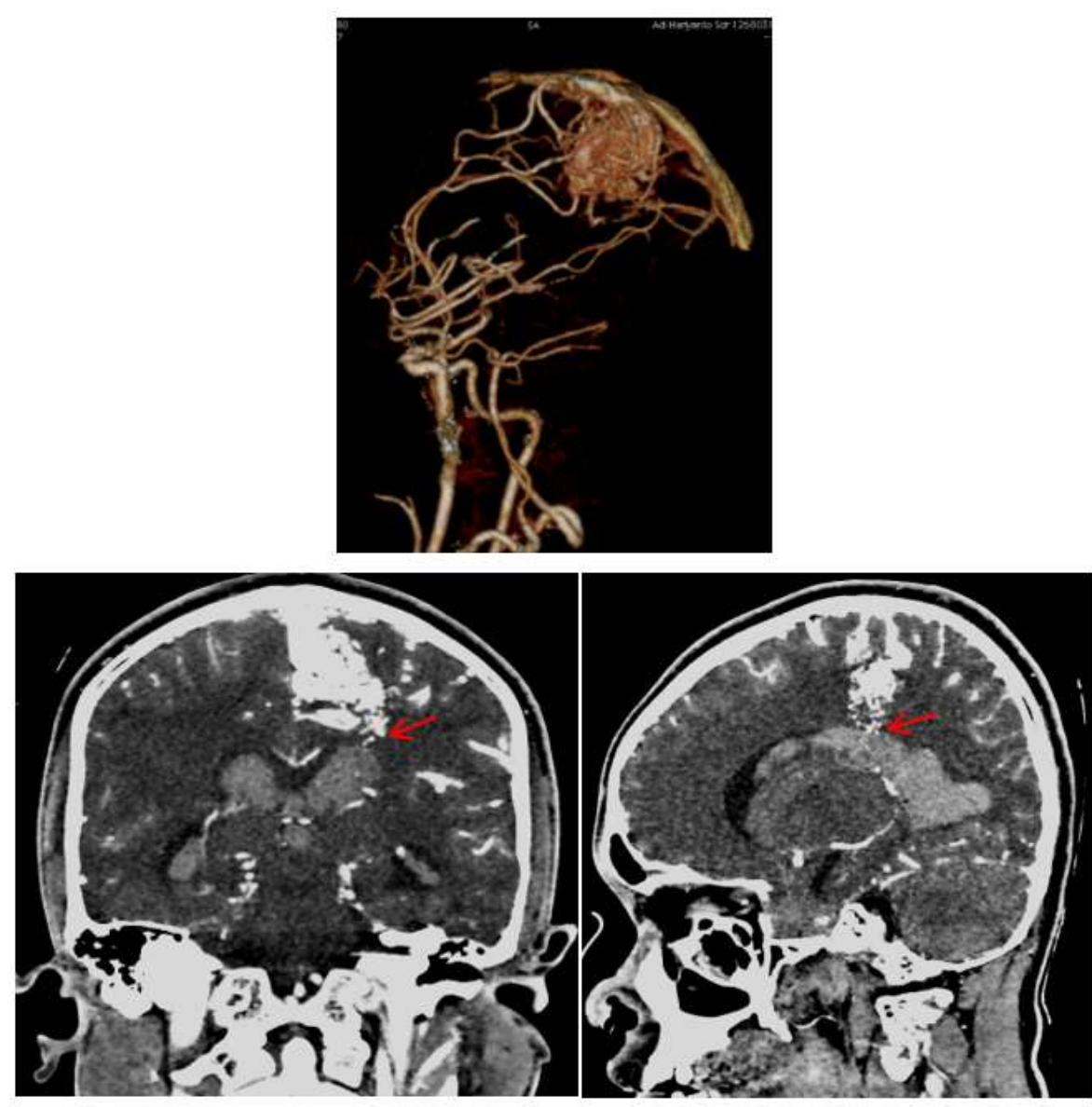

FIGURE 2: (A) Worm-like appearance image with multiple calcifications supporting an AVM in the left central semiovaleparietal cortex, and it receives a feeding artery from the left anterior cerebral artery branches (A2 and A3), draining veins direct to the superior sagital sinus and Trolard superior anastomotic vein (Fig. Spetzler-Martin Grade III Arteriovenous Malformation). (B) Image shows contact etween the nidus of the AVM and the ventricle on a CT scan of the head without contrast coronal view (C) sagital view. 


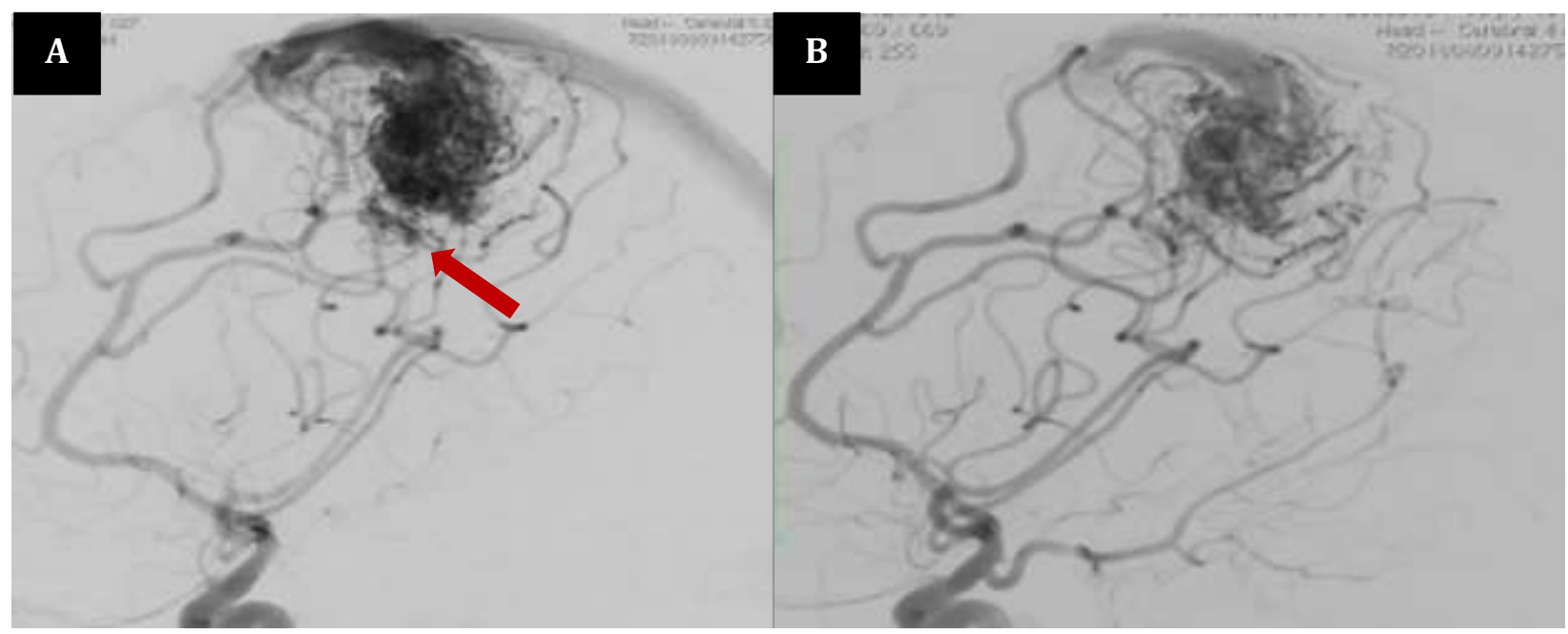

FIGURE 3: (A), Cerebral DSA lateral position image shows an intranidal aneurysm (red arrow) located at the edge of the lateral ventricle. (B) Cerebral DSA lateral position image after the embolization procedure. 